\title{
Gender Differences in Social Media Use and Cyberbullying in Belize: A Preliminary Report
}

\author{
Grace Mariko Kasahara ${ }^{1}$, Daniel Houlihan ${ }^{1} \&$ Collin Estrada ${ }^{2}$ \\ ${ }^{1}$ Minnesota State University, Mankato, USA \\ ${ }^{2}$ School Counselling and Care Unit, Ministry of Education, Youth and Sports, Belize \\ Correspondence: Daniel Houlihan, Minnesota State University, Mankato, USA
}

Received: March 5, 2019

Accepted: March 25, 2019

Online Published: March 26, 2019

doi:10.5539/ijps.v11n2p32

URL: https://doi.org/10.5539/ijps.v11n2p32

\begin{abstract}
Cyberbullying is a global issue that usually occurs with increased social media usage. A number of studies have found significant gender differences in social media use and cyberbullying, although gender differences are not consistent across studies. Despite the increase in access to cellphones and Internet in Belize, no studies have investigated how adolescents are using these resources. The purpose of this study was to investigate gender differences involving how adolescents in Belize are using social media and if cyberbullying is a problem.
\end{abstract}

Results provide preliminary information about social media use and cyberbullying in Belize. It indicates that gender differences exist within social media use and cyberbully reporting, and future studies should investigate influencing cultural factors.

Keywords: Belize, cyberbullying, gender differences, social media

\section{Introduction}

\subsection{Globalization of Cyberbullying}

Cyberbullying is a relatively recent phenomenon that occurs concomitant with increased social media usage in countries throughout the world (Sakellariou, Carroll, \& Houghton, 2012).

Numerous incidents of cyberbullying have been reported in countries such as the United States, Australia, Taiwan, South Africa, Turkey, and Mexico (Aricak et al., 2008; Burton \& Mutongwizo, 2009; Gámez-Guadix, Villa-George, \& Calvete, 2014; Huang \& Chou, 2010; Lenhart, 2007; Sakellariou et al., 2012). In 2006, an international review of 14 studies conducted in Canada, Australia, the United States, and the United Kingdom found that between 10 to 42 percent of participants were victims of some form of cyberbullying (Kraft, 2006). Most of the research studies about cyberbullying exist in Europe, North America, Australia, and Asia, while no studies have been conducted in South America despite the increase in cell phones and internet access in recent years.

\subsection{Defining Cyberbullying}

Jung et al. (2014) briefly defined cyberbullying as repeatedly inflicting harm to another person through the use of electronic devices. A longer definition was provided by Bill Belsey who stated that cyberbullying “...involves the use of information and communication technologies such as email, cellphone and text messages, instant messaging, defamatory personal websites, and defamatory online personal polling websites to support deliberate, repeated and hostile behavior by an individual or group that is intended to harm others (Burton \& Mutongwizo, 2009, p. 1).” Cyberbullies use a number of different sites and means of communication to attack their victims, which is important to keep in mind when conducting research on it.

\subsection{Negative Effects of Cyberbullying}

The negative effects of cyberbullying have been investigated by a number of studies and can be severe. Cyberbullying victims have been shown to have higher depressive symptoms, decreased self-esteem, academic problems, and increased suicidal ideation (Faryadi, 2011; Udris, 2014). The relationship between cyberbullying and depression is not culture-bound with studies finding analogous results in Spain, Korea, Mexico, and the United States (Gámez-Guadix, Orue, Smith, \& Calvete, 2013; Gámez-Guadix et al., 2014; Jung et al., 2014; Selkie, Kota, Chan, \& Moreno, 2015). Additionally, some studies have indicated that the negative 
effects of cyberbullying may vary based on the gender of the person being bullied (Bauman, Toomey, \& Walker, 2013). A study in Bauman et al. (2013) found that cyberbullying victimization was a significant predictor of depression for female high school students but not for males. Being a cyberbully was also noted as a significant predictor of suicide attempts for males but not for females. Given these differences, it is important to not only study cyberbullying but also how it is affecting genders differently.

\subsection{The Current State of Social Media Bullying}

Research on cyberbullying has been conducted in a number of countries throughout the world. A lot of studies have been conducted in the United States where $92 \%$ of adolescents' report that they go online daily and $71 \%$ have more than one type of social media account (Lenhart, 2015). Various studies have indicated that anywhere from $3 \%$ to $72 \%$ of students in the United States have been a victim of some form of cyberbullying (Selkie, Fales, \& Moreno, 2016). The large difference in estimated prevalence is most likely due to differences in research designs and parameters (e.g., different definitions of cyberbullying, age groups, etc.). One study in 2007 used a nationally representative sample of 935 teenagers and found that $32 \%$ had been a victim of some form of cyberbullying (Lenhart, 2007). This estimated prevalence may be more accurate because the study used a specific definition of cyberbullying and attempted to get a representative sample of the population. Additionally, while most of the research focuses on students from elementary school through high school, another study by MacDonald and Roberts-Pittman (2010) found that $22 \%$ of college students have also been cyberbullied during college. This suggests that cyberbullying affects a wide range of age groups in the United States.

Studies in the United States have also shown that there are gender differences in both social media usage and cyberbullying. A large study conducted in 2015 found that Instagram and Snapchat are dominated by girls in the United States with $61 \%$ of girls using Instagram compared to $44 \%$ of boys and $51 \%$ of girls using Snapchat compared to $31 \%$ of boys (Lenhart, 2015). Videogames are more male oriented with $84 \%$ of boys playing video games online compared to 59\% of girls. Another study conducted in 2013 found that $60 \%$ of adolescents have a private Facebook profile, which means that they only allow their friends to see their profile (Madden et al., 2013). This was significantly different by gender with more girls $(70 \%)$ having a private profile compared to boys $(50 \%)$. These gender differences in social media usage and communication may also affect gender differences in cyberbullying in the United States. One study found that a higher percentage of girls (38\%) reported being a victim of cyberbullying than boys (26\%; Lenhart, 2007). These gender differences may be cultural therefore it is important to investigate cyberbullying trends in other countries.

Australia has also conducted a number of studies on cyberbullying. A study in 2012 investigated cyberbullying among 1,530 male primary and secondary school students (Sakellariou et al., 2012). It found that $11.5 \%$ of the students had experienced cyberbullying during the school year. This is on the lower end of the prevalence estimates for cyberbullying in other countries, however, this study only included male students. Higher prevalence results were found in another study that included both male and female primary and secondary students in Australia (Dooley, Gradinger, Strohmeier, Cross, \& Spiel, 2010). That study found that $20 \%$ of the students had been a victim of cyberbullying with more girls (11\%) being a victim than boys $(9 \%)$. The overall estimates of prevalence in Australia are lower than some of those in the United States, however, a similar trend of more females being victims of cyberbullying was found.

Cyberbullying has also been a reported problem in countries in Asia. In Taiwan, a study in 2010 found that $35 \%$ of the junior high school students surveyed had been victims of cyberbullying (Huang \& Chou, 2010). The gender differences results found the opposite trend of western countries with more male students reporting experiences of being a victim of cyberbullying than females. Another study conducted in Korea found a much lower cyberbullying prevalence (3\%) among elementary and middle school students, but it found a similar trend in gender differences (Jung et al., 2014). Specifically, the results found that significantly more males (14\%) were involved in cyberbullying than females (5\%). It appears that more males are victims of cyberbullying but more males are also the perpetrators. This information suggests that gender differences in cyberbullying are influenced by culture, although it is unclear exactly what aspects of culture are driving these differences.

To our knowledge, no studies on cyberbullying and social media usage have been conducted in Central or South America, however, a study on cyberbullying was conducted on adolescents in Mexico in 2014 and it is a country that borders Belize (Gámez-Guadix et al., 2014). This study found a high prevalence rate of cyberbullying with $42 \%$ of adolescents reporting that they had performed a cyberbullying behavior and $45 \%$ reporting that they had been a victim of cyberbullying. Significantly more boys (47\%) than girls $(39 \%)$ had performed a cyberbullying behavior and more boys (47\%) than girls (45\%) were victims of cyberbullying although that was not a significant 
difference. These results relate more to the gender difference trends found in Asia, and the overall high prevalence of cyberbullying suggests this is a problem that needs to be addressed in Mexico.

Alarmingly, some studies have found that students are unwilling to report that they have been a victim of cyberbullying or that they know a victim of cyberbullying to an adult. A study conducted in the United States in 2008 found that $90 \%$ of the surveyed 12 to 17 year-olds reported that they did not tell an adult about cyberbullying incidents (Juvonen \& Gross, 2008). Another study in the United States found that the majority of the middle and high school students did not believe that adults at their school could help them if they were being cyberbullied (Agatston, Kowalski, \& Limber, 2007). The students were more likely to report being a victim of cyberbullying to a parent, however, a number of students were afraid that their parents would remove online privileges.

A study conducted in Canada investigated whether there were gender differences in whether or not a student would report being a victim of cyberbullying ( $\mathrm{Li}, 2006)$. The study found that no significant gender differences existed on whether students believed that adults could help stop cyberbullying. However, it found that females were significantly more likely than males to report being a cyber victim to adults. Another study conducted in Australia and Austria also found significant gender differences (Dooley et al., 2010). They found that about 90\% of girls had asked an adult for help with cyberbullying compared to $79 \%$ of boys in Australia. Additionally, a similar pattern was found in Austria with significantly more girls (42\%) compared to boys (19\%) seeking help. This indicates that gender differences in reporting cyberbullying may exist in other countries as well.

\subsection{The Current Study}

To our knowledge, no studies have been conducted on social media use and cyberbullying in Belize, despite the fact that Belize has seen a rise in technology and internet usage in recent years. The number of cellphone users in Belize increased from 7\% to 61\% from 2000 to 2016, and the reported percentage of people using the internet in 2016 was 45\% (Belize Profile, 2016; Mobile Cellular, 2015). These recent changes in technology usage mean that it is important to get information about how it is being used especially by younger people. Collecting information about gender differences in social media usage and cyberbullying can help officials in Belize understand if cyberbullying is a problem and how it is connected to social media usage.

The purpose of this study is to investigate whether there are gender differences in social media use and cyberbullying in Belize. Our first hypothesis is that there will be significant gender differences in the social media sites that Belize youth are using. Based on previous research in the United States, we hypothesize that Snapchat and Instagram will have more female users and gaming consoles will be used more by males.

Our second hypothesis is that significant gender differences will exist with social media privacy. Since females in the United States are more likely to prevent someone from accessing their Facebook profile content, we expect that significantly more females will keep social media content private in Belize.

Our third hypothesis is that we will find significant gender differences in cyberbullying. Since we found mixed results on whether more males or females will report being a victim of cyberbullying, it is unclear whether we should expect more females or males to report being a victim of cyberbullying in Belize. However, previous research does support our hypothesis that more females will be willing to report cyberbullying incidents to an adult.

\section{Method}

\subsection{Participants}

The participants in this study were recruited from primary and secondary schools in the six districts of Belize. Of the 303 participant responses, 68 were males and 235 were females between the ages of 11 to 25 years old $(M=14.77, S D=2.07)$. The students above the age of 18 were still included in this study because they were still in the secondary school environment. Most of the participants were from the Belize district (73\%), while the rest of the participants were from Cayo (14\%) and Toledo (13\%).

\subsection{Sampling Procedure}

Counselors with the Ministry of Education in Belize disseminated the surveys to willing participants after obtaining both the child and the parent's consent. The counselors were instructed to contact all of the students in primary and secondary schools in their school districts, so the schools were selected using convenience sampling. Surveys were administered in the child's classroom during a break period in the school day. In order to keep the survey information private, envelopes were given with the survey so students could conceal their answers after 
completion. Additionally, no identifying information was collected on the surveys in order to keep the participants anonymous. Participants were not compensated for taking the survey.

\subsection{Sample Size}

The sample size for both genders was intended to be equal, however, this study had a much larger number of female respondents compared to males. A chi-square test of independence was still used to assess gender differences because the assumptions of independence and expected frequencies were not violated for most of the questions. If the cells had an expected count of less than five, the fisher's exact test was referenced. However, caution should still be used to interpret the results because of the uneven sample sizes and because the data was obtained using convenience sampling.

\subsection{Measures}

A written 25-question survey was used to collect the data for this study. The survey took about 10 minutes to complete and it consisted of ranking items, rating items on a 5-point scale, yes or no questions, and questions that asked them to check all that apply. The survey asked them a few demographic questions (age, district, year in school, and gender) and then it asked them about how much time they spend on social media sites, what devices they used to access these sites, and their experiences with cyberbullying.

All of the counselors received the same written instructions about disseminating the survey to the participants, and they were able to contact the head researcher if they had any questions. This is the first social media and cyberbullying survey administered in Belize, so it does not have previously established cultural validity. However, it is based on previously conducted social media and cyberbullying surveys in Canada and Mexico (Gámez-Guadix et al., 2014; Li, 2006).

\subsection{Research Design}

Counselors first contacted the parents of students in order to obtain consent. Once all parents at a school had been contacted, counselors set up a time with school for the surveys to be distributed. Students were given an envelope containing an assent form and the social media survey by their school counselor during a break period in their school day. The students were instructed to review the assent form and if they wished to participate in the study, they had to sign it. Students had approximately 15 minutes to complete the assent form and survey. If the students did not want to participate, they were instructed to remain in their seats until the 15-minute survey-taking period was complete. After approximately 15 minutes elapsed, the students were told to place the survey and assent form, completed or not, back into the envelope. The students sealed the envelopes and return them to the counselors, who then mailed them to Daniel Houlihan Ph.D. at Minnesota State University, Mankato.

\subsection{Data Analysis}

Demographic information was collected on grade level, gender, age, and districts of Belize, however, grade level was removed from the analysis because the majority of the participants' responses to grade level were missing. The question may not have been clear enough to obtain accurate answers. Both gender and age were only missing about $1 \%$ of the data and the districts variable was missing about $13 \%$ of the data.

All of the relevant questions were checked for out-of-range responses and inaccurate responding. Many of the respondents seemed confused by the ranked questions that asked them to rank items from most to least. Respondents with odd responses (e.g., indicating that all items were the most used) were removed, which caused some of the ranked questions to have over $50 \%$ of the data counted as missing. This resulted in the removing of 4 out of the 5 ranked questions from the analysis. The only ranked question that remained was about responses to bullying on social media because all of the items on that question were missing less than $20 \%$ of the data. None of the other questions had a large problem with odd responding or missing data.

Since this study used a new survey, internal consistency reliability was measured on the questions that used rating scales ( $1=$ Very Often to $5=$ Never). It found an acceptable Cronbach's Alpha of .76 for the questions related to how often students are posting to different social media sites. An acceptable Cronbach's Alpha of .73 was also found for the questions about how often students are talking to social media friends outside of social media.

\section{Results}

\subsection{Social Media Sites}

Frequencies were run to gain percentages for the data. Facebook (75\%) had the largest percentage of students using it daily while Twitter had the lowest percentage (7\%). Additionally, 19\% of students reported that they post to Facebook very often compared to only $2 \%$ posting very often to Twitter. However, Snapchat (20\%) and 
other social media sites $(28 \%)$ both had higher percentages of students posting very often to their sites. These results can be seen in Table 1 .

Table 1. Social Media Usage

\begin{tabular}{lcc}
\hline Social Media Site & Percent Using Daily & Percent Posting Very Often \\
\hline Facebook & $75 \%$ & $19 \%$ \\
Snapchat & $34 \%$ & $20 \%$ \\
Twitter & $7 \%$ & $2 \%$ \\
Instagram & $31 \%$ & $13 \%$ \\
Kik & $13 \%$ & $8 \%$ \\
Confession Sites & $8 \%$ & N/A \\
Other & $38 \%$ & $28 \%$ \\
\hline
\end{tabular}

\subsubsection{Gender Differences for Social Media Sites}

Instagram and Snapchat both had significant gender differences in usage. According to a chi-square test of independence, there was a significant relationship between a student's gender and how often they post material on Instagram, $\chi 2(4)=14.95, p<.01$. As seen in Table 2, males were more likely to report never posting (64\%) than females $(39 \%)$, and females were more likely to report posting very often (16\%) than males $(5 \%)$. A similar significant relationship was found for Snapchat, $\chi 2(4)=13.78, p<.01$. Males were more likely to report never posting $(70 \%)$ than females $(44 \%)$, and females were more likely to report posting very often (24\%) than males (7\%). These results can be seen in Table 3.

Table 2. Percentages for Gender and Posting on Instagram

\begin{tabular}{|c|c|c|c|c|c|}
\hline \multirow[t]{2}{*}{ Gender } & \multicolumn{5}{|c|}{ Frequency of Posting on Instagram } \\
\hline & Never & $\underline{\text { Rarely }}$ & $\underline{\text { Sometimes }}$ & Often & Very Often \\
\hline Male & $64 \%$ & $10 \%$ & $17 \%$ & $3 \%$ & $5 \%$ \\
\hline Female & $39 \%$ & $19 \%$ & $16 \%$ & $11 \%$ & $16 \%$ \\
\hline \multicolumn{6}{|c|}{ Table 3. Percentages for Gender and Posting on Snapchat } \\
\hline \multirow[t]{2}{*}{ Gender } & \multicolumn{5}{|c|}{ Frequency of Posting on Instagram } \\
\hline & Never & $\underline{\text { Rarely }}$ & $\underline{\text { Sometimes }}$ & $\underline{\text { Often }}$ & $\underline{\text { Very Often }}$ \\
\hline Male & $70 \%$ & $9 \%$ & $7 \%$ & $7 \%$ & $7 \%$ \\
\hline Female & $44 \%$ & $12 \%$ & $10 \%$ & $11 \%$ & $24 \%$ \\
\hline
\end{tabular}

\subsection{Electronic Devices}

The results of this study indicated that $67 \%$ of students used smartphones, $43 \%$ used computers, and $41 \%$ used an iPad or tablet device to access the web. Students reported using smartphones the most with an average of 4.18 $(S D=2.00)$ hours per day, and gaming consoles were used the least with an average of $2.18(S D=1.74)$ hours per day (Table B4). Additionally, smartphones were used to access social media an average of $3.79(S D=2.03)$ hours per day, while gaming consoles were only used an average of $2.05(S D=1.65)$ hours per day (Table 4).

Table 4. Electronic Device Usage per Day

\begin{tabular}{lcc}
\hline Electronic Device & Average Hours Used & Average Hours on Social Media \\
\hline Smartphone & $4.18(S D=2.00)$ & $3.79(S D=2.03)$ \\
Computer & $2.82(S D=1.74)$ & $2.76(S D=1.82)$ \\
iPad/Tablet Device & $3.08(S D=2.00)$ & $2.90(S D=1.99)$ \\
Gaming Console & $2.18(S D=1.74)$ & $2.05(S D=1.65)$ \\
\hline
\end{tabular}




\subsubsection{Gender Differences in Electronic Devices}

According to a chi-square test of independence, there was a significant relationship between a student's gender and how many hours a day they spend using a gaming console, $\chi^{2}(6)=14.79, p<.05$. Seven of the cells had an expected count of less than five, therefore, the fisher's exact test was referenced and it found that the relationship was also significant $(p=.01)$. As seen in Table 5, the percentage of males $(16 \%)$ using a gaming console six hours a day was twice as high as females $(8 \%)$. Additionally, females $(65 \%)$ were more likely to report using a gaming console one hour a day than males (34\%).

Table 5. Percentages for Gender and Hours Spent on a Gaming Console per Day

\begin{tabular}{lcccccc}
\hline Gender & \multicolumn{5}{c}{ Hours Spend on a Gaming Console } \\
\hline Male & $\underline{2}$ & $\underline{3}$ & $\underline{3}$ & $\underline{4}$ & $\underline{5}$ & $\underline{6}$ \\
Female & $34 \%$ & $16 \%$ & $16 \%$ & $11 \%$ & $8 \%$ & $16 \%$ \\
\hline
\end{tabular}

\subsection{Social Media Privacy}

Students were also asked to report if they prevent certain people from accessing their social media content. $76 \%$ reported that they prevent certain people from accessing posts, while $24 \%$ reported that they did not. Teachers were prevented the most out of all types of people with $49 \%$ of students reporting that they prevent their teachers from accessing their social media content. Friends were prevented the least with only $22 \%$ of students reporting that they prevent a friend (Table 6).

Table 6. Percent of Students that Prevent Access from a Specific Type of Person

\begin{tabular}{lcc}
\hline Type of Person & Prevent Access to Social Media Content \\
\hline & $\underline{\text { No }}$ & $\underline{\text { Yes }}$ \\
Boss & $83 \%$ & $17 \%$ \\
Teachers & $51 \%$ & $49 \%$ \\
Parents/Guardians & $54 \%$ & $47 \%$ \\
Relatives & $58 \%$ & $42 \%$ \\
Friends & $78 \%$ & $22 \%$ \\
Other & $57 \%$ & $43 \%$ \\
\hline
\end{tabular}

\subsubsection{Gender Differences in Social Media Privacy}

A chi-square test of independence found that there was a significant relationship between a student's gender and whether they prevent certain people from accessing their social media content, $\chi^{2}(1)=5.67, p=.02$. As seen in Table 7, females were more likely to prevent certain people from accessing their social media content $(79 \%)$ than males $(64 \%)$.

Table 7. Percentages for Gender and Preventing Access to Social Media Content

\begin{tabular}{lcc}
\hline Gender & \multicolumn{2}{c}{ Prevents Access to Social Media Content } \\
\hline & $\underline{\text { No }}$ & $\underline{\text { Yes }}$ \\
Male & $36 \%$ & $64 \%$ \\
Female & $21 \%$ & $79 \%$ \\
\hline
\end{tabular}

3.4 Cyberbullying

Relating to cyberbullying, $15 \%$ of students reported being bullied on a social media site. This varied by district with $14 \%$ of the students being cyberbullied in Belize, $10 \%$ in Toledo, and $24 \%$ in Cayo. Overall, $66 \%$ of the surveyed students reported that they had a friend who has been bullied on a social media site. $72 \%$ of students also reported knowing someone who had bullied others while on social media, and $62 \%$ of students reported that cyberbullying was a problem in their school. Students ranked their responses to bullying on a scale of 1 to 4 ( $1=$ most, $4=$ least). They reported that they would most likely respond to bullying on social media by ignoring it $(M=2.00, S D=1.08)$, reporting it $(M=2.03, S D=1.06)$, and blocking it $(M=2.05, S D=1.03)$. They were least likely to participate in it $(M=3.74, S D=0.74)$. 


\subsubsection{Gender Differences in Cyberbullying}

According to a chi-square test of independence, there was not a significant gender difference for whether a student experienced being bullied on a social media website, $\chi 2(1)=2.28, p=.10$. However, as can be seen in Table 8 , more males $(21 \%)$ reported being bullied on a social media site than females $(13 \%)$.

Table 8. Percentages for Gender and Experienced Bullying on Social Media

\begin{tabular}{lcc}
\hline Gender & \multicolumn{3}{c}{ Experienced Bullying on Social Media } \\
\hline & $\underline{\text { No }}$ & $\underline{\text { Yes }}$ \\
Male & $79 \%$ & $21 \%$ \\
Female & $87 \%$ & $13 \%$ \\
\hline
\end{tabular}

A chi-square test of independence found that there was a significant relationship between a student's gender and whether they know a friend who has been cyberbullied, $\chi^{2}(1)=4.93, p=.03$. Females were more likely to report knowing a friend who had been cyberbullied $(66 \%)$ than males $(50 \%)$. Additionally, there was a significant relationship between a student's gender and whether they know people who have cyberbullied others on social media sites, $\chi^{2}(1)=12.06, p=.001$. Females were also more likely to report knowing someone who cyberbullied others $(76 \%)$ than males $(54 \%)$. Similar significant results were found for the relationship between gender and whether students believe that cyberbullying is a problem in their school, $\chi^{2}(1)=6.29, p=.01$. Females were more likely to report that they think it is a problem $(65 \%)$ than males $(48 \%)$.

There was only one significant gender difference among the ranked responses to cyberbullying. A significant relationship was found between a student's gender and whether they would report cyberbullying, $\chi 2(3)=8.84$, $p=.03$. As seen in Table 9 , females were more likely to rank reporting cyberbullying as the action they would be most likely to do (48\%) than males $(26 \%)$.

Table 9. Percentages for Gender and Likelihood of Reporting Cyberbullying

\begin{tabular}{lcccc}
\hline Gender & \multicolumn{4}{c}{ Likelihood of Reporting Cyberbullying } \\
\hline Male & $\underline{2}$ & $\underline{3}$ & $\underline{4}$ (Least) \\
Female & $26 \%$ & $26 \%$ & $31 \%$ & $18 \%$ \\
\hline
\end{tabular}

Students were asked to rank their responses to cyberbullying on a scale from 1 to $4(1=$ most, $4=$ least $)$.

\section{Discussion}

The results of this study supported our first hypothesis of significant gender differences occurring within social media sites. Snapchat and Instagram both had significantly more females using those sites often, and males were spending significantly more time using gaming consoles. This is similar to the results found in the United States and suggests that social media sites in Belize are being used in a similar way.

Our second hypothesis was also supported by the results of this study. Similar to the United States, females were more likely than males to prevent certain people from accessing their social media content. The percentages were higher in Belize for both males and females, however, our study also asked a broader question about privacy of all social media content compared to the study in the United States that only asked about Facebook. It is possible that the percentages in our study would have been lower if the question only asked about Facebook privacy.

The third hypothesis in our study was only partially supported. We did not find significant gender differences for being a victim of cyberbullying. More males reported being a victim of cyberbullying compared to females, which is similar to the results found in Asia and Mexico. This indicates that some aspect of culture shared between countries in Asia and Belize may be influencing this gender difference. Additionally, the overall prevalence of cyberbullying in Belize (15\%) was much lower than the prevalence in Mexico (42\%; Gámez-Guadix et al., 2014). This is surprising because the countries are so close to each other and they share a similar trend in gender differences. However, this difference may exist because of the differences between the Belize and Mexico samples. 84\% of the students in the Mexico study had access to cellphones that they could use to communicate, compared to $67 \%$ of the students in our sample that had access to smartphones. Additionally, $94 \%$ of the students in the Mexico sample used the Internet compared to the estimated prevalence of $45 \%$ of the population of Belize having access to the Internet (Belize Profile, 2016). This means that as cell phone and internet access increase in Belize, the number of students being cyberbullied may increase as well. 
An interesting and unexpected result of this study occurred with the gender differences in the other cyberbullying questions. Despite the results indicating that more males are cyberbullying victims, females were significantly more likely to know a friend who has been cyberbullied. Females were also significantly more likely to know a cyberbully and think that cyberbullying is a problem at their school. This gender difference may be occurring because of communication differences between genders. Students may be more willing to talk about cyberbullying with female students and this would result in more female students being aware that cyberbullying is occurring at their school.

Our third hypothesis also predicted that more females would report cyberbullying incidents to an adult compared to males. This hypothesis was supported by the results with significantly more females reporting that they would most likely respond to cyberbullying by reporting it. The results of our study are similar to the results found in Austria but much lower than the results found in Australia (Dooley et al., 2010). It is possible that students in Australia feel like they can trust adults at their school to help with cyberbullying compared to students in other countries. Studying how Australia handles cyberbullying in schools could help increase the number of students seeking help in other countries.

\subsection{Limitations}

While this study provides a lot of preliminary information about social media usage and cyberbullying in Belize, there are a number of limitations that should be considered. This study used convenience sampling so the results might not generalize to the adolescent population in Belize. Additionally, surveys were sent to all of the districts of Belize and responses were received from three of the six districts. Most of the responses came from the Belize District, and this also limits the generalizability of the study.

Another limitation of this study is the large difference between the number of males and females in this study. In order to protect confidentiality, no specific school information was collected, however, it is possible that some of the schools that were surveyed were female only schools. Another possibility is that females were more willing to take an optional survey during school time. It is unclear exactly why the responses were overwhelmingly from females, but for the most part it did not affect the ability of the chi-square tests of independence to detect significant differences. When there was an issue of expected cell counts of less than five, the fisher's exact test was referenced. This test helps correct this problem, however, those results should still be considered with caution.

The survey in this study also has some limitations. While it is based on questions used in previous studies, it has not been evaluated for validity or test-retest reliability with the current population. The survey also may have missed some of the commonly used social media sites in Belize because $38 \%$ of the students selected "Other" when asked what social media sites they use daily.

\subsection{Implications for Future Research and Conclusion}

The results of this study can provide a good basis for future studies conducted in Belize and possibly other countries in South America. It can be used by officials in Belize to help formulate a plan to address cyberbullying in each school district and for each gender. Future replications of this study should re-evaluate the ranked questions on the survey and possibly include an example of how ranked questions should be answered. Additionally, future researchers could include an initial verbal explanation of how to answer ranked questions and the grade level question. A new question that should be included in a replicated version of this study should be about whether the participant has cyberbullied other people. This would give us an idea about how many students are self-reported cyberbullies and if a significant gender difference exists.

Further research also needs to be conducted to assess validity and test-retest reliability of this survey and use a better sampling procedure. While convenience sampling provided preliminary information about cyberbullying in Belize, it is unclear if this result can generalize to the overall population. It is also important for future studies to get samples from all of the districts of Belize and get a sample sizes that are more similar between genders.

An interesting study that could be conducted in the future should look at cultural factors influencing gender differences in cyberbullying. It looks like some cultural factors may be causing more males to be victims of cyberbullying in countries in Asia and Belize. Multiple studies in North America and Europe indicate that females prefer indirect and verbal aggression, which might be why cyberbullying is more female oriented (McAndrew, 2014). However, studies in Asia and South America have also found similar trends with girls preferring relational aggression and boys preferring overt aggression (Cheng, 2009; Hines \& Fry, 1994; Huang \& Chou, 2010). Therefore, it is still unclear why there are similar trends in aggression across cultures, but there are different gender differences in cyberbullying. Other cultural factors may be influencing this difference. 
In conclusion, this is the first study to provide information about social media usage and the prevalence of cyberbullying in Belize. It also provides evidence that gender is influencing social media usage and some aspects of cyberbullying. Cyberbullying has already started among students in Belize and future studies need to monitor the changes overtime.

\section{References}

Agatston, P. W., Kowalski, R., \& Limber, S. (2007). Students' Perspectives on Cyber Bullying. Journal of Adolescent Health, 41(6), S59-S60. https://doi.org/10.1016/j.jadohealth.2007.09.003

Aricak, T., Siyahhan, S., Uzunhasanoglu, A., Saribeyoglu, S., Ciplak, S., Yilmaz, N., \& Memmedov, C. (2008). Cyberbullying among Turkish adolescents. Cyberpsychology \& Behavior, 11(3), 253-261. https://doi.org/10.1089/cpb.2007.0016

Barlett, C. P., Gentile, D. A., Anderson, C. A., Suzuki, K., Sakamoto, A., Yamaoka, A., \& Katsura, R. (2014). Cross-Cultural Differences in Cyberbullying Behavior: A Short-Term Longitudinal Study. Journal of Cross-Cultural Psychology, 45(2), 300-313. https://doi.org/10.1177/0022022113504622

Bauman, S., Toomey, R. B., \& Walker, J. L. (2013). Associations Among Bullying, Cyberbullying, and Suicide in high school students. Journal of Adolescence, 36(2), 341-350. https://doi.org/10.1016/j.adolescence.2012.12.001

Belize Profile, 2016. Belize Profile (Latest data available: 2016). Retrieved from https://www.itu.int/net4/itud/icteye/CountryProfileReport.aspx?countryID=38

Burton, P., \& Mutongwizo, T. (2009). Inescapable Violence: Cyber Bullying and Electronic Violence Against Young People in South Africa. Centre for Justice and Crime Prevention, 8, 1-12.

Cheng, C. L. (2009). No Blood Means Less Harm?: Relational Aggression and Peer Rejection in Adolescence. Journal of Educational Psychology, 40(3), 511-528.

Dooley, J. J., Gradinger, P., Strohmeier, D., Cross, D., \& Spiel, C. (2010). Cyber-Victimisation: The Association Between Help-Seeking Behaviours and Self-Reported Emotional Symptoms in Australia and Austria. Journal of Psychologists and Counsellors in Schools, 20(2), 194-209. https://doi.org/10.1375/ajgc.20.2.194

Faryadi, Q. (2011). Cyber Bullying and Academic Performance. International Journal of Computational Engineering Research, 1(1), 23-30.

Gámez-Guadix, M., Orue, I., Smith, P. K., \& Calvete, E. (2013). Longitudinal and Reciprocal Relations of Cyberbullying with Depression, Substance Use, and Problematic Internet Use Among Adolescents. Journal of Adolescent Health, 53(4), 446-452. https://doi.org/10.1016/j.jadohealth.2013.03.030

Gámez-Guadix, M., Villa-George, F., \& Calvete, E. (2014). Psychometric Properties of the Cyberbullying Questionnaire (CBQ) Among Mexican Adolescents. Violence and victims, 29(2), 232-247. https://doi.org/10.1891/0886-6708.vv-d-12-00163r1

Hines, N. J., \& Fry, D. P. (1994). Indirect Modes of Aggression Among Women of Buenos Aires, Argentina. Sex Roles, 30(3-4), 213-236. https://doi.org/10.1007/bf01420991

Huang, Y. Y., \& Chou, C. (2010). An Analysis of Multiple Factors of Cyberbullying Among Junior High School Students in Taiwan. Computers in Human Behavior, 26(6), 1581-1590. https://doi.org/10.1016/j.chb.2010.06.005

Jung, Y. E., Leventhal, B., Kim, Y. S., Park, T. W., Lee, S. H., Lee, M., ... \& Park, J. I. (2014). Cyberbullying, Problematic Internet Use, and Psychopathologic Symptoms Among Korean Youth. Yonsei Medical Journal, 55(3), 826-830. https://doi.org/10.3349/ymj.2014.55.3.826

Juvonen, J., \& Gross, E. F. (2008). Extending the School Grounds?-Bullying Experiences in Cyberspace. Journal of School Health, 78(9), 496-505. https://doi.org/10.1111/j.1746-1561.2008.00335.x

Kraft, E. (2006). Cyberbullying: A Worldwide Trend of Misusing Technology to Harass Others. WIT Transactions on Information and Communication Technologies, 36, 155-166. https://doi.org/10.2495/is060161

Lenhart, A. (2015). Teens, Social Media \& Technology Overview 2015. Pew Research Center, 9.

Lenhart, A. (2007). Cyberbullying and Online Teens. Pew Research Center.

Li, Q. (2006). Cyberbullying in Schools: A Research of Gender Differences. School Psychology International, 27(2), 157-170. https://doi.org/10.1177/0143034306064547 
MacDonald, C. D., \& Roberts-Pittman, B. (2010). Cyberbullying Among College Students: Prevalence and Demographic Differences. Procedia-Social and Behavioral Sciences, 9, 2003-2009. https://doi.org/10.1016/j.sbspro.2010.12.436

Madden, M., Lenhart, A., Cortesi, S., Gasser, U., Duggan, M., Smith, A., \& Beaton, M. (2013). Teens, Social Media, and Privacy. Pew Research Center, 21, 2-86.

McAndrew, F. T. (2014). The "Sword of a Woman": Gossip and Female Aggression. Aggression and Violent Behavior, 19(3), 196-199. https://doi.org/10.1016/j.avb.2014.04.006

Mobile Cellular, 2015. Retrieved from https:/www.itu.int/en/ITUD/.../2015/ Mobile_cellular_2000-2014.xls

Sakellariou, T., Carroll, A., \& Houghton, S. (2012). Rates of Cyber Victimization and Bullying Among Male Australian Primary and High School Students. School Psychology International, 33(5), 533-549. https://doi.org/10.1177/0143034311430374

Selkie, E. M., Fales, J. L., \& Moreno, M. A. (2016). Cyberbullying Prevalence Among US Middle and High School-Aged Adolescents: A Systematic Review and Quality Assessment. Journal of Adolescent Health, 58(2), 125-133. https://doi.org/10.1016/j.jadohealth.2015.09.026

Selkie, E. M., Kota, R., Chan, Y. F., \& Moreno, M. (2015). Cyberbullying, Depression, and Problem Alcohol Use in Female College Students: A Multisite Study. Cyberpsychology, Behavior, and Social Networking, 18(2), 79-86. https://doi.org/10.1089/cyber.2014.0371

Udris, R. (2014). Cyberbullying Among High School Students in Japan: Development and Validation of the Online Disinhibition Scale. Computers in Human Behavior, 41, $253-261$. https://doi.org/10.1016/j.chb.2014.09.036

\section{Copyrights}

Copyright for this article is retained by the author(s), with first publication rights granted to the journal.

This is an open-access article distributed under the terms and conditions of the Creative Commons Attribution license (http://creativecommons.org/licenses/by/4.0/). 